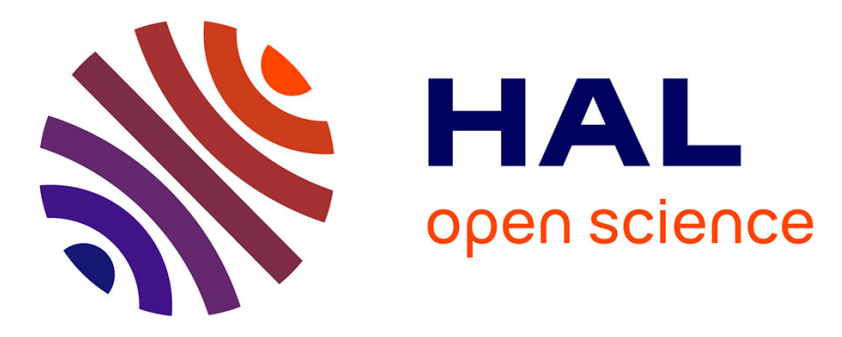

\title{
Adaptation of the GreenLab model for analyzing sink-source relationships in Chinese Pine saplings
}

\author{
Hong Guo, Veronique Letort, Lingxia Hong, Thierry Fourcaud, Paul-Henry
}

Cournède, Yuanchang Lu, Philippe de Reffye

\section{- To cite this version:}

Hong Guo, Veronique Letort, Lingxia Hong, Thierry Fourcaud, Paul-Henry Cournède, et al.. Adaptation of the GreenLab model for analyzing sink-source relationships in Chinese Pine saplings. PMA06, Plant growth modeling, Simulation, Visualization and Applications, Nov 2006, Beijing, China. pp.236243. hal-00576583

\section{HAL Id: hal-00576583 https://hal.science/hal-00576583}

Submitted on 14 Mar 2011

HAL is a multi-disciplinary open access archive for the deposit and dissemination of scientific research documents, whether they are published or not. The documents may come from teaching and research institutions in France or abroad, or from public or private research centers.
L'archive ouverte pluridisciplinaire HAL, est destinée au dépôt et à la diffusion de documents scientifiques de niveau recherche, publiés ou non, émanant des établissements d'enseignement et de recherche français ou étrangers, des laboratoires publics ou privés. 


\title{
Adaptation of the GreenLab model for analyzing sink-source relationships in Chinese Pine saplings
}

\author{
Guo Hong ${ }^{1 *}$, Letort Veronique ${ }^{2}$, Hong Lingxia ${ }^{1}$, Fourcaud Thierry ${ }^{3}$, Cournede Paul-Henry ${ }^{2}$, \\ Lu Yuanchang ${ }^{1}$, De Reffye Philippe ${ }^{3,4}$ \\ 1 CAF, Research Institute of Forest Resource Information Techniques, Beijing, 100091 China \\ 2 Ecole Centrale Paris, Applied Mathematics Laboratory, Chatenay-Malabry, 92290 France \\ 3 CIRAD, UMR AMAP, Montpellier, F-34000 France \\ 4 INRIA, DigiPlante, Rocquencourt, F-78150 France
}

\begin{abstract}
Since the 1990s, a new generation of models has emerged to simulate tree growth with consideration of both tree structure and functional processes. However, calibration of these functional-structural models (FSMs) often remains an open problem due to the topological complexity of trees and to the heavy measurements required. In this paper, we explore a possible way for dealing with the fitting problem, based on the GreenLab model approach.

Detailed organ-level data including topological and geometrical measurements were collected on eight Pe Pine saplings (Pinus tabulaeformis carr.) E, near Beijing. Adaptation of GreenLab to introduce a flexible modeling for biomass allocation to ring growth is presented. The main assumptions, such as allometry rules and sink relationships, were investigated. The problem of calibration of a complex branching structure was solved by defining an average tree. The results were interpreted with particular focus on the ones concerning the hidden mechanisms of secondary growth.
\end{abstract}

\section{troduction}

Pinus tabulaeformis Carr., also called Chinese pine, is the most widespread temperate coniferous species [1] and grows in over 10 provinces in China. As it is one of the most important ornamental conifers used for reafforestation, many studies have been carried out on this species, including forest management, ecology and genetics aspects. Mathematical simulation of individual growth was developed with structure information [2]. In most studies, the biomass of an individual tree, the biomass of stands and its net primary productivity were considered on the basis of statistical analysis of measurement data $[3,4]$. However, considering both structure and biomass points of view can help to understand the growth process and biomass allocation of this species. For example, to simulate key processes such as light interception [5][6] or competition for resources (light, water, nutriments) inter and intra trees, it is crucial to take into account the tree branching architecture. This is why functional-structural models have recently emerged [7]. They aim at combining organogenesis and photosynthesis, organs playing their true roles as sources and sinks for biomass during the development process. However, validation and calibration of these models remains an open problem and often relies on qualitative observations or on global scale variables such has crown diameter or total biomass. Moreover, the large stochastic variability of branching patterns of a same species in given environmental conditions is a major obstacle to fit the precise architecture of a tree. For example, the LIGNUM model [8] was parameterized on young Scott Pines and Jack Pine using parameter values from bibliography or estimated by fitting global scale variables and the validation was done only on height and trunk diameter. The EMILION model [9] simulates very precisely physiological processes such as maintenance and growth respiration or biomass fluxes at the organ level for maritime pines but the parameterization of the corresponding equations are taken from the literature and are not necessarily available for other species. Although this kind of approach provides powerful methods to analyze the inside processes such as the causes of axes death, it is not suitable for integration in support decision systems for forest management, since data concerning micro- 
climate and geometrical shape at organ level are almost impossible to get for a large number of individuals.

In this paper, a simplified approach of tree simulation is presented and illustrated on eight young Chinese pines. This approach relies on the GreenLab model [10] that was developed in LIAMA (Laboratory of Computer Sciences, Automation and Applied Mathematics, Beijing, China) and that is oriented to be a functional-structural model for agronomy and forestry applications [11]. Its main characteristic is to extract some relevant assumptions to simplify the tree growth simulation and calibration, thus opening the way to deal with tree complexity [12]. In agronomy, it has been calibrated on many crops such as maize [13], sunflower [14] or tomato [15]. These examples illustrate the generic property of the model but they all deal with plants with simple architecture. However, thanks to its automaton-based organogenesis process [16], GreenLab can simulate complex architectures as well and the use of a substructure factorization algorithm [12] speeds up dramatically the simulation, which is a key point for any fitting process at whole tree level. In recent developments of the model, influence of photosynthesis on organogenesis has been introduced [17, 18], allowing realistic simulations of tree growth depending on a global environmental factor. In our preliminary study, only the deterministic version of the model was used to fit the source-sink balance of the Chinese pine saplings, that is to say neither stochastic nor environment-driven variations were included.

In the way to extend the application area of the GreenLab model to forestry field, particular attention was given to secondary growth modelling, which is the kernel of any model aiming at simulating tree growth. A pioneer work was realized by Mitchell et al. [19] on Douglas-fir: they took into account crown competition through their projected surface and stipulated that radial growth increment is proportional to the number of leaves above the ring position in the tree. Then Houllier et al. [20] adapted that model to spruce (CEP). The LIGNUM model incorporates the pipe theory [21] to relate the thickness increment in the main stem to the above living foliage. Recently, some attempts have been made to bridge the gap between process-based models and structural models that output global relationships between radial growth and crown size [22, 23]. Deleuze and Houllier [24] used a reactiondiffusion model for radial growth but it was not related to the crown structure and to the assimilate allocation to the whole tree. In this paper, by referring to those concepts, a new way to simulate secondary growth is introduced: first, biomass is allocated to a global pool for rings, depending on the current vigor state of the tree, and then this biomass is distributed all over the tree with respect to its architecture through an extension of the Pressler law. This method allows overcoming the often-stated limitations of the widely used Pressler law [24].

This paper presents an adaptation of GreenLab to explore the possibilities of tree source-sink calibration. We tested and partially validated some of the basis hypotheses of the model such as allometric relationships and constant sinks on the Chinese pine saplings.

\section{Model and main hypotheses}

\subsection{Organogenesis based on botanical rules}

Studies on tree architecture were initiated in 1978 by the botanists Francis Hallé et al. [25] who defined 23 architectural models that represent tree development. Plants can be considered as a set of basic botanical units, called metamers, made of one internode and axillary buds that give birth to leaves, fruits and axes. Pine tree growth is rhythmic with a period of one year, called growth cycle (GC), which is the time step of the model: all growth variables are integrated at this time scale. The number of growth cycles since the appearance of an organ or an axis is called its chronological age (CA). The succession of metamers appearing during one growth cycle is called a growth unit $(\mathrm{GU})$. Axes are sorted into categories according to their physiological age (PA), which are defined based on the differentiation states of their apical meristem. Each class is defined by the structure of its GU (acrotony, fruit presence...), as presented in Barthélémy and Caraglio [26]. All axes of same PA have the same set of parameters, driving allometry and functioning rules.

In the analysis of Chinese pine, for sake of simplicity, a GU was regarded as a single virtual metamer and the needles in one GU were considered as a single virtual leaf. The axis PA was defined according to its branching order: axis of PA 1 (the main stem) bear axes of PA 2, axes of PA 2 bear axes of PA 3 and so on.

\subsection{Biomass production and allocation}

Biomass production and allocation are calculated at each GC based on sink-source relationships between the organs in the plant. The sources include biomass from the seed (available at the first cycles only) and the production of biomass by the leaves through 
photosynthesis. This production is set up based on the transpiration law of plant and an empiric relationship adapted from Beer-Lambert law to simulate the effects of self-shading in an isolated plant [27]. The amount of biomass $Q(i)$ generated by the green needle surface $S(i)$ at GC $i$ can be expressed by Eqn (1):

$$
Q(i)=\frac{E(i) \cdot S_{p}}{r}\left(1-\exp \left(-k \cdot \frac{S(i)}{S_{p}}\right)\right)(1) .
$$

$r$ is defined as the hydraulic resistance of the plant to transpiration [13], $E(i)$ is the average potential environmental factor during $G C i . S_{p}$ is the total ground projection area available for the plant, as defined in [28], and $k$ is a parameter taking into account leaf inclination.

GreenLab assumes that this biomass is gathered in a common pool and then allocated to the living organs (internodes, needles, rings, and fruits) that compete according to their sink strength to get it, regardless to their position on the tree. The total demand of the plant consists of two parts: demand for buds and demand for ring compartment $D_{r g}(i)$. For an organ $o$ of PA $k$, with sink strength $P_{o}$, the biomass acquisition $\Delta q_{o}(k, i)$ at $\mathrm{GC} i$ can be described as:

$$
\Delta q_{o}(k, i)=P_{o}(k) \cdot \frac{Q(i-1)}{\sum_{o \in\{a, e\}} P_{o}(k) N_{o}(k, i)+D_{r g}(i)}
$$

whith $Q(i-1)$ the total biomass production at GC $i-1$, $D_{r g}(i)$ the demand of rings at $\mathrm{GC} i$ and where $o$ represents indices for organ types (needles: $a$; internodes: $e) . N_{o}(k, i)$ is the number of organs belonging to category $o$ at GC $i$ and PA $k$. For trees, a major assumption of the model is that the organ expansion can be considered as immediate since it lasts less than one GC and is of short duration compared to the GC duration (one year). Thus this sink strength can be regarded as constant, which implies that the ratio between newly created needles and internodes should be constant.

\subsection{Secondary growth}

In standard conditions, it has been observed that tree primary growth in height take precedence over their secondary growth [29]. To simulate this behaviour, it is assumed that the ring compartment can play a buffer role: its demand $D_{r g}$ depends on available supply and demand of biomass at GC $i$ :

$$
D_{r g}(i)=P_{O}+P_{1} \cdot \frac{Q(i-1)}{D(i)}
$$

where $P_{0}$ is the constant sink (dimensionless) of the rings compartment and $P_{l}$ is the corresponding slope per mass unit $\left(\mathrm{g}^{-1}\right) . D(i)$ is the total demand of the plant at $\mathrm{GC} i$.

Biomass allocated to the ring pool $Q_{r g}$ is proportional to its demand and to the ratio Q/D. From this pool, biomass is partitioned to the ring of each internode individually, through two possible modes: the common pool mode and the Pressler [30] mode.

In the common pool mode, biomass for all the rings in the whole tree at GC $i$ goes to a common pool and then reallocation to each new ring is done according to its PA $k$ and to its length $l(k, i)$. It is represented as:

$$
D_{\text {pool }}(i)=\sum_{k=1}^{P A_{m}} N_{i}(k, i) \cdot p_{r g}(k) \cdot l(k, i)
$$

where $P A_{m}$ is the maximal $\mathrm{PA}$ in the tree, $l(k, i)$ is the length of the $N_{i}(k, i)$ internodes of PA $k$ on plant of CA $i$ and $p_{r g}(k)$ is the linear density of sink of rings for internodes of PA $k$. In the Pressler mode, the biomass going to each ring is proportional to the number of leaves above it in the branching structure [31]. It means that each leaf gives its biomass production to a "pipe" [21] running until the bottom of the tree and the demand of this pipe is proportional to its length. With the same notations, it can be expressed as in Eqn (5):

$$
D_{\text {pressler }}(i)=\sum_{k=1}^{P A_{m}} \sum_{p \in P_{k}} N_{a}(k, i, p) \cdot p_{r g}(k) \cdot l(k, i)
$$

where $N_{a}(k, i, p)$ is the number of leaves at GC $i$ above the considered internode at PA $k$ and whose position in the tree architecture is represented by $p . P_{k}$ is the set of possible positions $p$ of metamers of PA $k$ in the whole plant of age $i$. It has been reported in some cases [24][32] that the Pressler rule is affected by environmental changes or throughout the tree growth and that it does not represent buttress formation. So a parameter $\lambda(0 \leq \lambda \leq 1)$ allows mixing the two modes together, assuming that they can have combined effects with different degrees in different species during biomass partitioning to each new ring. Thus the biomass $q_{r g}$ given to the new ring of an internode of PA $k$ and position $p$ at $\mathrm{GC} i$ is:

$q_{r g}(k, i, p)=\left(\frac{1-\lambda}{D_{p o o l}(i)}+\frac{\lambda N_{a}(k, i, p)}{D_{\text {pressler }}(i)}\right) \cdot p_{r g}(k) \cdot l(k, i) \cdot Q_{r g}(i)$

(6) where the parameter definitions are the same than in Eqn (3)-Eqn(5).

\subsection{Allometries for organ shape}

Since, the model variables are biomass increments, the organ shape is derived from its current mass using simple allometric rules. The fresh matter of an internode consists of two parts: the pith (primary growth) and the rings (secondary growth). The pith is 
regarded as a perfect cylinder and the relationship giving the length $l(k, i)$ from the pith mass at GC $i$ and PA $k$ is estimated as in Guo et al. [13].

$$
l(k, i)=\sqrt{b_{e}(k)} \cdot q_{e}(k, i)^{\frac{1+\beta}{2}}
$$

where $q_{e}(k, i)$ is the fresh matter available at GC $i$ for the internode of PA $k$ and $b_{e}$ and $\beta$ are shape coefficients which are fitted from measurements of the GU lengths and fresh masses, assuming here that pith wood density is constant (Figure 1). For secondary growth, the thickness of the new ring of a GU depends on the allocated quantity of biomass to build it: a new layer is added on the initial cylinder.

Concerning needle active surface area, the functioning of the needles of one $\mathrm{GU}$ is considered to be the same as the one of a single virtual leaf whose thickness $\varepsilon$ is constant. The leaf surface area is related to leaf mass $q_{a}$ according to Yang and Yang [33]:

$$
S_{a}=12.4176 \cdot q_{a}
$$

\section{Data Analysis}

\subsection{Tree materials}

The eight Chinese pine sapling's data included two trees of one-year-old, two-year-old, three-year-old trees and five-year-old respectively. They were taken from the nursery garden which is open area in Shisanling forest farm, Beijing $\left(39^{\circ} 50^{\prime} \mathrm{N}, 116^{\circ} 25^{\prime} \mathrm{E}\right)$ in the spring of 2006 .

In these experiments, the generative organs were not considered. For each metamer, GU length and GU diameter, needle fresh mass and internode fresh mass were measured. The needles functioning time [13] was set to 3 GC since no needles were found on GU older than 3 years.

\subsection{Parameters estimated from data}

Concerning our Chinese pine saplings, several types of input parameters can be calculated directly from the measurements.

3.2.1. Internode Allometry. From the measurement of fresh mass and length of internodes located at tips of axes of each PA (when secondary growth can be considered as negligible), the parameters $b_{e}$ and $\beta$ needed in Eqn.7 can be estimated for each PA (Figure 1). The detailed results can be seen in Table 1 (logarithmic scale). The $\mathrm{R}^{2}$ coefficient ranges from 0.76 to 0.94 , confirming the choice for the relationship between internode mass and internode length for Chinese Pine saplings.

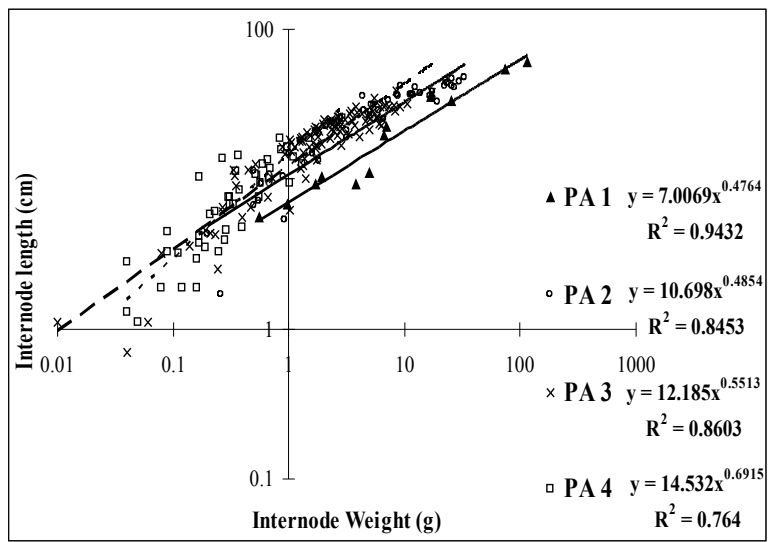

Figure1. Internode Allometry

3.2.2. Internode and needle sinks. As explained in the first part, assuming that needles and internodes on the newly created tips of axes expand immediately implies that their sink ratios can be measured directly. It means that there should be a simple proportionality between internode and needle mass on the newly created GU (Eqn. 2), the proportionality ratio being the sink value. Thus the sink of an organ o of PA $k$ is estimated as in Eqn (11):

$$
P_{o}(k)=\frac{\overline{q_{o}}(k, i)}{\overline{q_{a}}(1, i)}
$$

where $\bar{q}_{o}(\mathrm{k}, \mathrm{i})$ is the average mass of organ o of PA $\mathrm{k}$ that appeared at last $\mathrm{GC} \mathrm{i}$ (the tip axes one) and where $\bar{q}_{a}(1, \mathrm{i})$ is the average needles fresh mass on the tip of the main stem. The sink of the needles on the main stem (PA 1) is the reference and is arbitrarily set to 1 so sinks for all the other organs with different PA are relative values. The sink values are gathered in Table 1.

Table1. Sinks and Allometric coefficients

\begin{tabular}{llcccc}
\hline \multirow{2}{*}{ Name } & \multirow{2}{*}{ Meaning } & \multicolumn{4}{c}{ Values } \\
\cline { 3 - 6 } & & PA1 & PA2 & PA3 & PA4 \\
\hline$P_{e}$ & Internode Sink & 0.80 & 0.38 & 0.11 & 0.02 \\
$P_{a}$ & Blade Sink & 1.00 & 0.71 & 0.28 & 0.07 \\
$b_{e}$ & $\begin{array}{l}\text { Internode } \\
\text { allometry }\end{array}$ & 62 & 127 & 148 & 211 \\
$\beta$ & Internode & -0.01 & -0.12 & 0.1 & 0.38 \\
& $\begin{array}{l}\text { allometry } \\
\varepsilon\end{array}$ & Needles SLW & & \multicolumn{2}{c}{0.08 g.cm $^{-2}$} \\
\hline
\end{tabular}


To test the assumption of constant sink ratios, the needle mass is drawn with regard to internode mass of the same GU (Figure 2). It reveals that there is a good linear relationship between needle mass and internode mass of GU of axis tips for each PA $\left(\mathrm{R}^{2}\right.$ coefficient ranges from 0.7 to 0.93 ). It implies that the assumption of constant sink ratios is reasonable.
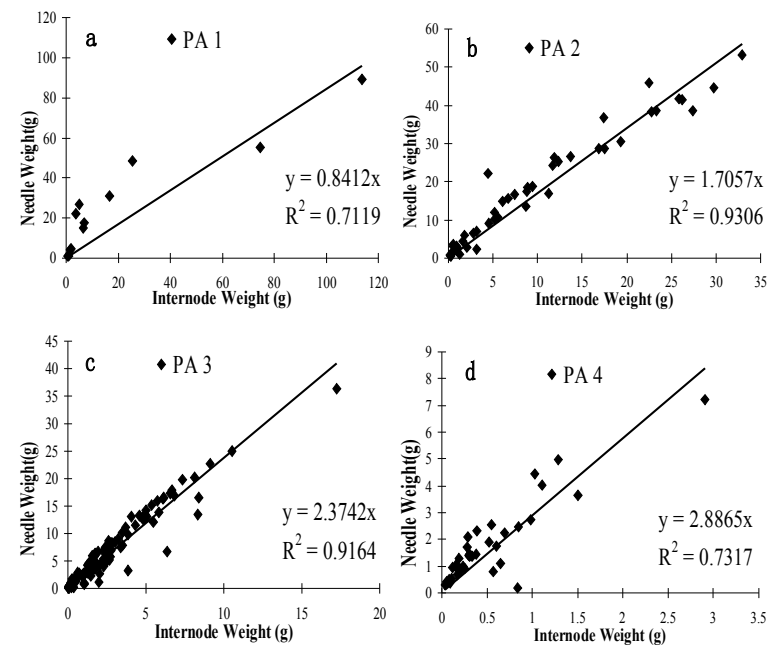

Figure 2. Internodes and needles mass ratio

Moreover, from the slope coefficients of figure 2, it can be observed that the average ratio of needle mass over internode mass increases with PA of the GU. It means that for Chinese pine saplings, the allocation to internodes decreases with branching order to the benefit of needles.

\section{Hidden parameters: fitting methods and results}

\subsection{Tree topology}

For each tree, an average topology (axes numbers per whorl, GU numbers per axis) was defined according to the substructure factorization algorithm implemented in GreenLab to speed up simulation. The algorithm consists of a multi-scale factorization of the parts that replicate several times in the branching system. It is based on the botanical observations given in part 2.1 and [26]. It reduces drastically the simulation time - since each structure having given physiological and chronological ages is computed only once - but still allows realistic outputs.

Concerning the functional data, metamer fresh weights (needles and internodes) were averaged for each axis type and length and diameter were kept from the measured axis that was the closest from the average one. Thus a target file could be built in the same way as described in Guo et al. [13]. The axes were identified by their PA and CA. They were described GU by GU with internode weight, length and diameter and needle weight. The target files included the eight trees. They were gathered in categories based on their axes numbers on the main stem so that it could be assumed that trees with the same topology were in fact repetitions of the same tree at different ages. Thus it can be considered that the target file includes data from only three trees at different growth stages.

\subsection{Hidden parameters: calibration of the model}

The hidden parameters of GreenLab are more difficult to calibrate since they are all linked through the dynamical mechanisms of the model and each of them can have a complex influence on all the outputs. So, even if the data concerns different types of organs and are of different ranges of orders, all of them have to be considered together in the fitting procedure. For the eight young trees, the target files included about 60 items of GU data. A same set of parameters should fit dynamically the three growth trajectories, since the trees were assumed to share the same species-specific parameters and to differ only on their topological structure and biomass seeds. To solve this inverse problem [34], the nonlinear generalized least square method was implemented in the Digiplante software [12].

For Chinese pine saplings, only five parameters remained to be fitted: the coefficient for needle resistance $r$, the sink strength of rings and the corresponding slope, $P_{0}$ and $P_{1}$, the parameter $\lambda$ driving the biomass repartition from the ring biomass pool and the linear density of sink of rings for internodes of PA 2. This sink linear density is found negligible for internodes of PA 3 and PA 4. Since there was very few axes overlapping on the measured young trees, $S_{p}$ had too little influence on estimating the $Q(i)$ in Eqn 1 and could be set to one. Calibration results are gathered in Table 2. Figure 3 shows the comparison between calibrated and measured data for some of the outputs, the axis scale being logarithmic whenever required by the large order range of the data. The fitting graphs represent cumulated internode (3a) and needle mass (3b) of the tree at each GC (from year 1 to year 5), internode mass (3c) and diameter (3d) GU by GU on the main stem, internode (3e) and needle (3f) mass on each $\mathrm{GU}$ of the branches for each PA. On figures $3 \mathrm{e}$ and $3 \mathrm{f}$, each $\mathrm{x}$ - abscissa represents one branch of the tree and each point of this abscissa corresponds to one 
simulated (filled symbols) or measured (empty symbols) GU of the branch. On several PA 3 branches, data are missing, especially for needle mass, and are yet simulated.

The fitting of the compartment data had priority on the organ-by-organ one since it was used to set the initial values of the parameters that have a global effect (the resistance and the parameters defining the ring demand). Thus the total biomass evolution of the trees was reasonably well fitted, even if it can be noticed that the first stages were under-estimated. Although the fitting on branches is not perfect, in particular due to the large variability of data, the global trend of organ mass variation according to their age and position in the tree is correctly reproduced.
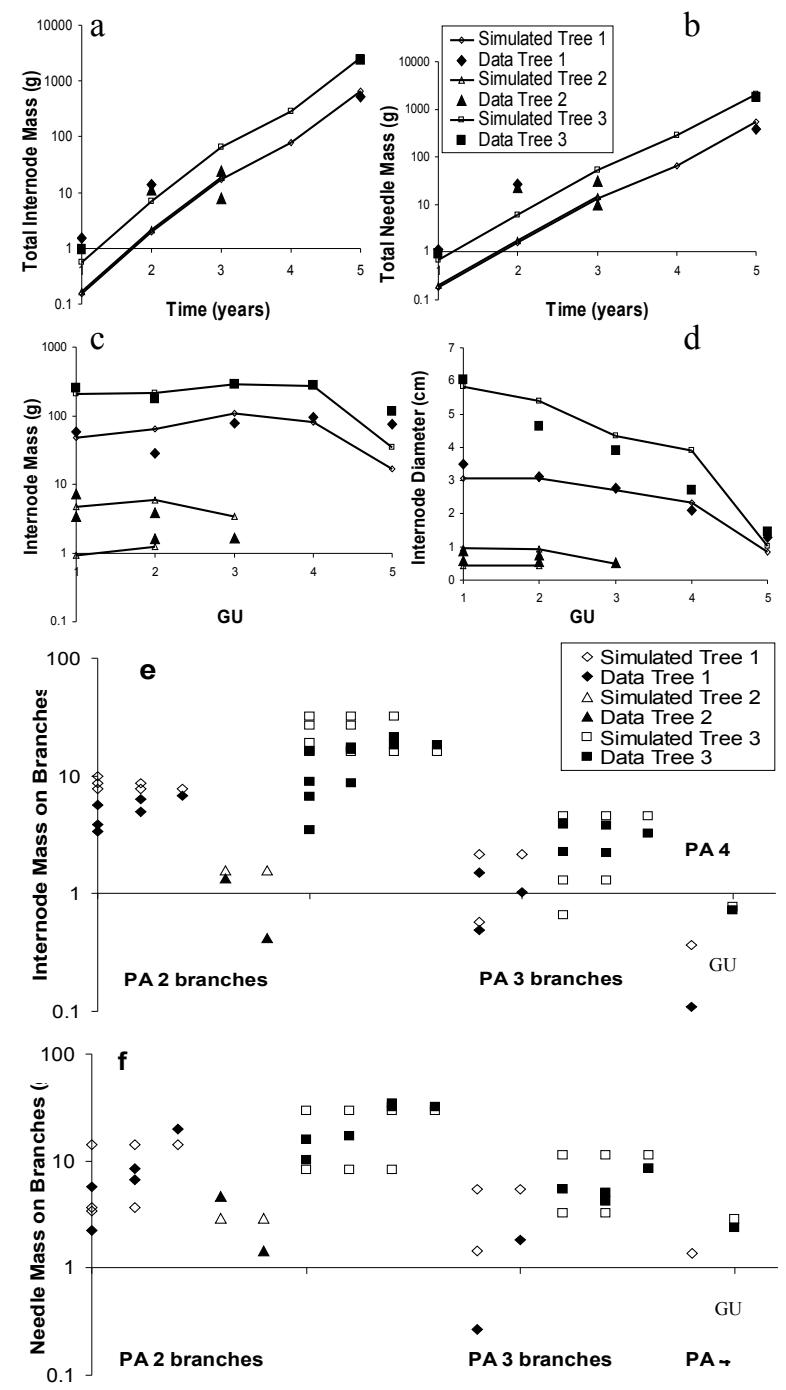

Figure 3. Multi-fitting results.
The same set of parameters allows reproducing the growth trajectory of the three trees and more particularly of two quite different five-year old trees, the cumulated internode mass of the strongest one being more than four times higher than the second one. The only difference between these two simulated trees is their seed biomass (ranging from 0.4 to $1.4 \mathrm{~g}$ ) and their axis numbers for each PA. This is sufficient to get strong differences in the evolution of their $\mathrm{Q} / \mathrm{D}$ ratio and thus to their radial growth.

Table 2. Hidden parameter value

\begin{tabular}{|c|c|c|c|}
\hline Name & Meaning & Value & $\begin{array}{l}C V(\% \\
\end{array}$ \\
\hline$r$ & Needles resistance & 0.64 & 3.5 \\
\hline$P_{0}$ & Sink for ring pool & $2.64 \mathrm{~g}$ & 47.5 \\
\hline$P_{1}$ & $\begin{array}{l}\text { Slope for ring sink } \\
\text { variation }\end{array}$ & 0.77 & 41.6 \\
\hline$\lambda$ & $\begin{array}{l}\text { Ring biomass } \\
\text { allocation parameter }\end{array}$ & 0.14 & 74.8 \\
\hline$p_{r g}(2)$ & $\begin{array}{c}\text { Linear density of PA } 2 \\
\text { ring sink }\end{array}$ & 0.11 & 39.7 \\
\hline
\end{tabular}

The parameters have high coefficient of variation values because the main sources of error are the topological structure and the sinks. Indeed, concerning rings, only data of five-year-old trees can help to discriminate the parameters. But our objective was to present a generic model so the parameters concerning biomass allocation to rings were kept. The fitting of the model allows estimating at the same time the biomass quantity allocated to secondary growth and the proportion of assimilate going up in the tree architecture, the rest of it being only authorized to be allocated in the part below the considered source. The small value of $\lambda(=0.14)$ found for the pine saplings shows that there is almost uniform reallocation of rings biomass, i.e. the localization of biomass sources does not influence a lot the allocation.

\subsubsection{Simulation and Visualization}

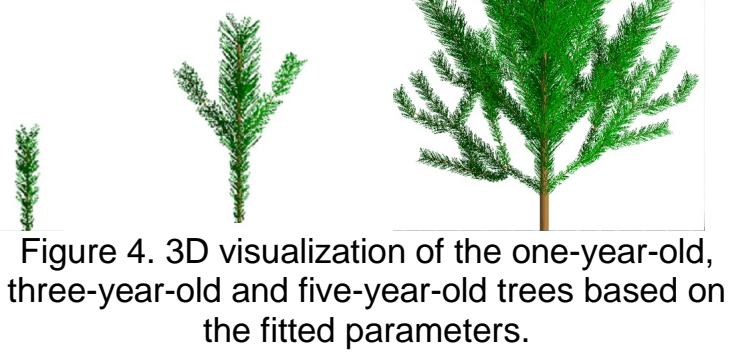


One purpose of the simulations with GreenLab model was to produce realistic Chinese pine saplings based on observation and measurement data. The calibrated parameters were used to simulate and visualize 3D representation of pine trees using the Digiplante software (Figure 4).

\section{Discussion}

Functional-structural model GreenLab has been previously tested on crops and other herbaceous but, even if tree simulation was already performing [12], it was the first attempt to calibrate it on tree functioning. The results on allometric rules revealed to be correct in view of the data on internodes (Figure 1). The assumption of immediate expansion, which implies a constant mass ratio between needles and internodes of a newly created GU, was in accordance with the data analysis (Figure 2). This hypothesis allows strong simplifications and is one of the bases of GreenLab. However, it needs to be tested on adult trees and to study whether shading and competitions have an effect on the sink ratios, which is highly probable [35]. Further study is required to detect possible evolution of sink values or relationship between sink strength of an organ and its position within the tree.

Concerning secondary growth, we presented a model for biomass allocation to a ring compartment that depends on the current trophic state of the tree (through the $\mathrm{Q} / \mathrm{D}$ ratio). Thus the resulting system is self- adaptive to environmental changes. The process of biomass partitioning in the tree structure is considered as an independent step. The introduction of parameter $\lambda$ to mix the Pressler mode and Pipe mode brings more flexibility to the model of biomass partitioning for rings but there is always an affine relationship between ring increment surface and number of leaves above its position, as soon as the parameter $\lambda$ is strictly positive. By calibrating the value of $\lambda$, the fitting procedure acts as a tool to analyze the allocation processes inside the trees. The value found was $\lambda=0.14$ for the Chinese saplings, which means there is almost uniform allocation of rings biomass. This results seems reasonable since the trees are still very young, but the fitting has few interest and cannot be considered as a validation of the model for the ring part, biomass allocation to ring being influent only in five year-old trees.

For saplings, the substructure factorization algorithm can seem unnecessary, but it allows simulating adult trees in a low computational time [17], which is a key condition to open access to any fitting procedure at organ level. However, the measurement protocol will need to be adapted to the structural complexity of older trees.

Further work still need to be done to complete this study and to deal with different environmental conditions and tree ages. However, this first work indicates that the basic hypotheses are relevant for Chinese pine trees. So this functional-structural model, based on botanical principles, has a good potential to describe tree growth and development.

\section{Acknowledgements}

The authors thank the students and the colleagues from CAF involved in collecting the measurement data. This study was funded by National Natural Science Foundation of China (Grant No. 60073007 ; $30230290 ;$ 60473110) and supported by Sino-French Laboratory for Computer Sciences, Automation and Applied Mathematics (LIAMA) in Beijing.

\section{References}

[1] T. X. Luo, W. H. Li and S. D. Zhao. "Productivity distribution patterns and modeling of Pinus tabulaeformis forest in china". Chinese Journal of Applied Ecology. 1999, 10(3): pp. 257-261.

[2] X.J. Han, "Mathematical simulation of individual tree growth of Pinus tabulaeformis". Journals of Beijing Forestry University. 1988, 10(1): pp.1-9.

[3] S. R. Dong and Y. X. Guan. "Studies on productivity of Pinus tabulaeformis stands in Taiyue mountain, Shanxi province". Journal of Beiijng Forestry University. 1980: pp.1-20.

[4] Q. Y. Ma. "A Study on the Biomass of Chinese Pine Forests". Journals of Beijing Forestry University, 1989, 10(11): pp.1-10.

[5] C. Soler, F. Sillion, F. Blaise, P. de Reffye, "An efficient instantiation algorithm for simulating radiant energy transfer in plant models". ACM Transactions on Graphics, 2003, 22(2): 204-233.

[6] H. Sinoquet, G. Sonohat, J. Phattaralerphong, C Godin, "Foliage randomness and light interception in 3-D digitized trees: an analysis from multiscale discretization of the canopy". Plant, cell and environment, 2005, 28, pp. 1158-1170.,

[7] Sievänen R, Nikinmaa E, Nygren P, Ozier-Lafontaine H, Perttunen J, Hakula H., "Components of functionalstructural tree models". Ann Sci 2000(57), pp. 399-412. 
[8] J. Perttunen, E. Nikinmaa, M. J. Lechowicz, R. Sievänen, C. Messier. "Application of the functional-structural tree model LIGNUM to sugar maple saplings (Acer saccharum Marsh) growing in forest gaps". Ann. Bot. 2001. 88 (3), 471-481.

[9] A. Bosc. "EMILION, a tree functional-structural model: presentation and first application to the analysis of branch carbon balance", Annals of Forest Science, 2000, 57(5/6): 555-570.

[10] P. de Reffye, M. Goursat, J. P. Quadrat and B. G. Hu. "The dynamic equations of the tree morphogenesis: GreenLab Model". Beijing, China: Tsinghua University Press/ Springer-Verlag, 2003, pp.108-117

[11] B. G. Hu, P. de Reffye, X. Zhao, H. P. Yan and M. Z. Kang. "GreenLab: A new Methodology towards Plant Functional-structure Model-Structural Aspect", Beijing, China: Tsinghua University Press/ Springer-Verlag, 2003, pp.21-35.

[12] P.H. Cournède, M. Z. Kang, A. Rostand-Mathieu, H. P. Yan, B. G. Hu and P. de Reffye. "Structural factorization of Plants to Compute their Functional and Architectural Growth". Simulation, 2006, 82(7): pp.427-438.

[13] Y. Guo, Y. Ma, Z. G. Zhan, B. Li, M. Dingkuhn, D. Luquet and P. de Reffye. "Parameter optimization and field validation of the functional-structural model GREENLAB for maize", Annals of Botany, 2006, 97(2): pp.217-230.

[14] Y. Guo, P. de Reffye,Y. H. Song,Z.G. Zhan,M. Dingkuhn, B.G. Li. "Modeling of biomass acquisition and partitioning in the architecture of sunflower".In: $\mathrm{Hu}$ B-G, Jaeger M eds. Beijing, China: Tsinghua University Press/ Springer-Verlag, 2003,pp. 271-284.

[15] Q. X. Dong, Y. M. Wang, J. F. Barczi, P. de Reffye and J. L. Hou. Tomato Growth Modeling Based on Interaction of its Structure-Function. Beijing, China: Tsinghua University Press/ Springer-Verlag, 2003, pp.250-262.

[16] X. Zhao, P. de Reffye, D. Barthélémy and B. G. Hu. "Interactive simulation of plant architecture based on dualscale automaton model", Beijing, China: Tsinghua University Press/ Springer-Verlag, 2003,pp.144-153

[17] A. Rostand-Mathieu, P. H.. Cournède, P. de Reffye 2004. "A dynamical model of plant growth with full retroaction between organogenesis and photosynthesis", ARIMA 4 : 101-107.

[18] A. Rostand-Mathieu. Essai sur la modélisation des interactions entre la croissance et le développement d'une plante : cas du modèle GreenLab. Paris, France: Ecole Centrale Paris, 2006.
[19] K. J. Mitchell. "Dynamics and simulated yield of Douglas-fir", Forest Science Mono graph, 1975, 21(4): 39.

[20] F. Houllier, J. M. Leban and F. Colin. "Linking growth modelling to timber quality assessment for Norway spruce", Forest Ecology and Management, 1995, 74: pp. 91-102.

[21] K. Shinozaki, K. Yoda, Hozumi, K and T. Kira. "A quantitative analysis of plant form - the pipe model theory I. Basic Analysis”, Japanese Journal of Ecology, 1964, 14(13): pp. 97-105.

[22] J. M. Ottorini, N. Le Goff and C. Cluzeau. "Relationships between crown dimensions and stem development in Fraxinus excelsior", Canadian Journal of Forest Research, 1996, 26: pp. 394-40.

[23] S. Pouderoux and J. F. Deleuze, C., Dhote. "Analysis of crown efficiency in a Common Beech thinning trial using a process-based model.", Annals of Forest Science, 2001, 58(3): pp.261-27.

[24] C. Deleuze and F. Houllier. "A flexible radial increment taper equation derived from a process-based carbon partitioning model", Annals of Forest Science, 2002, 59: pp.141-154.

[25] F. Hallé, R.A.A. Oldeman, P. B. Tomlinson. "Tropical trees and forests". Berlin, Heidelberg, New York: SpringerVerlag, 1978.

[26] D. Barthelemy and Y. Caraglio. "Plant architecture: a dynamic, multilevel and comprehensive approach to plant form, structure and ontogeny", Annals of Botany, 2007, 99(3): pp. 375-407.

[27] T.A. Howell and J.T. Musick, "Relationship of dry matter production of field crops to water consumption", Crop Water Requirements, 1985. pp. 247-269.

[28] J.W. Wilson, "Analysis of light interception by single plants", Annals of Botany, 1981, 48: pp. 501-505.

[29] R. M. Lanner. "On the insensivity of height growth to spacing", Forest Ecology and Management, 1985, 13: pp. 143-148.

[30] E. Assmann, F. Franz. Vorläufige Fichten-Ertragstafel für Bayern. Forstwis-senschaftliches Centralblatt 84 (1/2), Sonderdruck, Verlag Paul Parey, Hamburg/Berlin, (1965) S.1-68.

[31] D. A. White, "Relationships between foliar number and the cross-sectional areas of sapwood and annual rings in red oak (Quercus rubra) crowns", Canadian Journal of Forest Research. 1992 (23), pp. 1245-1251.

[32] D. Pont, "Analyses of basic crown-stem growth relationships in Radiata pine". Functional Structural Plant Models (FSPM04), 2004, pp. 105-109. 
[33] D. Yang, X. Q. Yang, "Studies on biomass and productivity of Pinus tabulaeformis plantation in the Wufengshang of Wudu, Gansu Province". Journal of Northwest Normal University Natural Science. 2004.(1), pp. 70-73.

[34] Z. G. Zhan, P. de Reffye, F. Houllier and B. G. Hu, "Fitting a structuralfunctional model with plant architectural data". Beijing, China: Tsinghua University Press/ SpringerVerlag, 2003, pp. 236-249

[35] U. Niinemets, D. S. Ellsworth, A. Lukjanova and M. Tobias, "Site fertility and the morphological and photosynthetic acclimatation of Pinus sylvestris needles to light.", Tree Physiology, 2001 (21), pp. 1231-1244. 